\title{
Ethnobotanical survey in Canhane village, district of Massingir, Mozambique: medicinal plants and traditional knowledge
}

\author{
Ana Ribeiro ${ }^{1 *}$, Maria M Romeiras' ${ }^{1}$, João Tavares', Maria T Faria²
}

\begin{abstract}
Background: Medicinal plants are used by $80 \%$ of people from developing countries to fulfill their primary health needs, occupying a key position on plant research and medicine. Taking into account that, besides their pharmaceutical importance, these plants contribute greatly to ecosystems' stability, a continuous documentation and preservation of traditional knowledge is a priority. The objective of this study was to organize a database of medicinal plants including their applications and associated procedures in Canhane village, district of Massingir, province of Gaza, Mozambique.

Methods: In order to gather information about indigenous medicinal plants and to maximize the collection of local knowledge, eleven informants were selected taking into account the dimension of the site and the fact that the vegetation presents a great homogeneity. The data were collected through intensive structured and semistructured interviews performed during field research. Taxonomical identification of plant species was based on field observations and herbarium collections.
\end{abstract}

Results: A total of 53 plant species have been reported, which were used to treat 50 different human health problems. More than half of the species were used for stomach and intestine related disturbances (including major diseases such as diarrhea and dysentery). Additionally, four species with therapeutic applications were reported for the first time, whose potential can further be exploited. The great majority of the identified species was also associated with beliefs and myths and/or used as food. In general, the community was conscientious and motivated about conservational issues and has adopted measures for the rational use of medicinal plants.

Conclusions: The ethnomedicinal use of plant species was documented in the Canhane village. The local community had a rich ethnobotanical knowledge and adopted sound management conservation practices. The data compiled in this study show the social importance of the surveyed plants being a contribution to the documentation of PGR at the national and regional level.

\section{Background}

In ancient times, medicinal plants have been used all over the world as unique sources of medicines and may constitute the most common human use of biodiversity $[1,2]$. According to the World Health Organization, $80 \%$ of people in developing countries still depend on local medicinal plants to fulfill their primary health needs [3]. Besides that, there is a global consensus on the benefits of phytopharmacy and at present medicinal plants

\footnotetext{
* Correspondence: aribeiro@itgb.unl.pt

'Tropical Research Institute, Rua da Junqueira 86, 1300-344, Lisbon, Portugal
} Full list of author information is available at the end of the article occupy a key position in plant research and medicine. These facts associated with the progressive loss of traditional knowledge, due to rural exodus, and with the threats to which Plant Genetic Resources (PGR) are exposed, make the efforts to study and preserve PGR relevant in every respect. In this context, several conservation studies have been performed [4-6].

Like most African countries, Mozambique is an important repository of biological diversity. This diversity is used by ca. $90 \%$ of the country's population to fulfill its housing, food, energy and health needs. According to [7], in Mozambique approximately 15\% of the total PGR (ca. 5,500 plant species) is used by rural

\section{C) Biomed Central}


communities for medical purposes and plays a key role in basic health care. Despite a long history of medicinal plants use in Mozambique, research on this subject is still incipient [8-10] and poorly disseminated, focusing mainly on medicinal plant markets and trade issues from Maputo province [7]. The work presented in this article reports on the utilization of medicinal plants in the Canhane village, district of Massingir, Province of Gaza. The last survey in the region dates from 1960-70 [11,12].

Canhane village is located $32^{\circ} 09^{\prime} 30^{\prime \prime} \mathrm{E}$ and $24^{\circ} 4^{\prime} 30^{\prime \prime}$ S (Figure 1). With an extension of 7,200 ha, the village has a flat landscape with slopes ranging from 0 to $2 \%$ and altitudes from $95 \mathrm{~m} \mathrm{~N}$ to $200 \mathrm{~m} \mathrm{~S}$ [13]. The climate is semi-arid with two seasons: (i) dry season (April/May to October/November), with temperatures varying from $14.5^{\circ} \mathrm{C}$ to $28.5^{\circ} \mathrm{C}$ and a maximum annual precipitation of $67.9 \mathrm{~mm}$; and (ii) hot and rainy season (October/ November to April/May), with temperatures ranging from $19.9^{\circ} \mathrm{C}$ to $32.8^{\circ} \mathrm{C}$ and a maximum annual precipitation of $370 \mathrm{~mm}$ [14]. The humidity index may vary between -50 and -70 , the negative values indicating the dryness of the region [15]. The soils are essentially sandy with a low to moderate percentage of organic matter (0-3\%) and thus poor for agriculture.

The village has 1357 inhabitants (51\% women, 49\% men) the great majority belonging to the Valoyi ("Witch doctor") family from the Changana ethnic group [16,17]. The community has poor access to water resources, health services (the closest health center is located in the Massingir village, seven $\mathrm{Km}$ away from Canhane), trading and communications, an obsolete energy system and an unsuccessful school system. Due to the lack of a

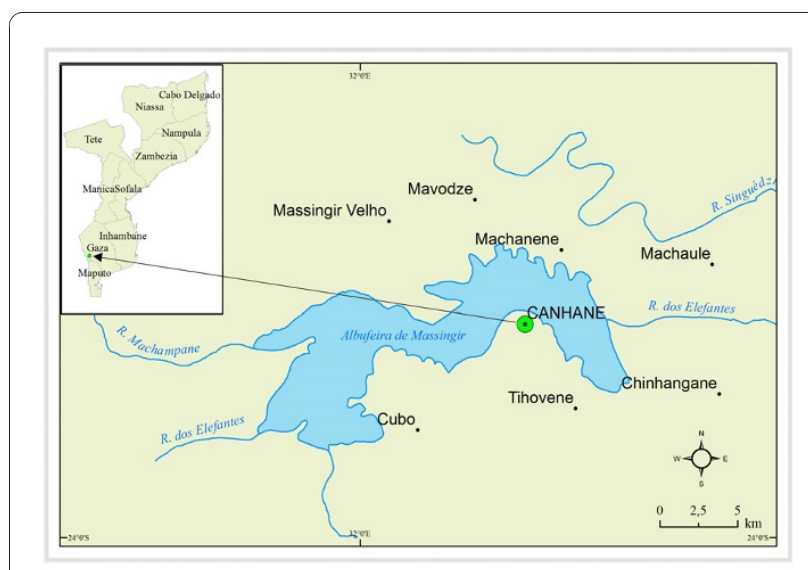

Figure 1 Geographical location of the study site. Left: Map of Mozambique illustrating the geographical position of the Province of Gaza and the District of Massingir. Right: Geographical position of Canhane within the District of Massingir. local health center, traditional medicine plays an important role in basic health care. The main activity is agriculture, followed by livestock and fisheries. Handicraft is a tertiary activity.

The major habitat types of Canhane are woodlands, savannah and grasslands $[18,19]$. Currently, the vegetation communities are at different levels of degradation mainly due to human practices (e.g. production of firewood, charcoal and grazing). The over-exploitation of resources and the limiting environmental conditions seem to be associated with the decay of the resilient capacity of the ecosystems as evidenced by the occurrence of great devastated areas [17].

With this study, we intended to contribute to the conservation and valorization of the local floristic and cultural heritage. It should be noted that the study area is of particular importance, since it is located in the heart of the Limpopo National Park, which together with Kruger National Park (South Africa) and Gonarezhou National Park (Zimbabwe) constitute the Great Limpopo Transfrontier Park and Conservation Area (GLTP). The study reports on 53 medicinal plant species and their traditional applications.

\section{Methods}

\section{Ethnobotanical data collection}

The work was initiated with a meeting between the researchers, the community leader and the Commission for Social Management from Canhane Village, in order to: i) explain the aim and importance of the work and its integration on the Community-based Development Program; ii) get cooperation and permission to use the cultural heritage; iii) collect information for structuring the interviews; iv) give orientations for the selection of informants by age and gender; and v) plan the field activities.

Eleven informants (six men and five women) were selected as the best traditional knowledge holders. The selection criteria were based on the size of the study site, the vegetation homogeneity and on the indications provided by the community.

Due to reasons related to beliefs and myths, it was not possible to get the information directly from Witch doctors. However, it should be highlighted that most of the Canhane inhabitants belong to the Valoyi ("Witch doctor") family. Using standard methods [20,21], the data was collected through intensive structured interviews and complemented with semi-structured interviews in local language (i.e. Changana). These included: common and local name of the plant, applications, parts of the plant used, methods of preparation and administration routes. Translation to Portuguese was validated by linguistic specialists. 


\section{Taxonomic identification}

The medicinal plants reported by the informants were collected during three field surveys (in October of 2007 and in March and November of 2008). The team was accompanied by two local guides with a deep knowledge of local flora. Species identification was done during the field visits and by comparing voucher specimens with specimens deposited at the Herbarium of the Faculty of Sciences, Universidade Eduardo Mondlane (LMU, Maputo, Mozambique). The scientific names were confirmed through specialized bibliography [22-25] as well as the African Plant Database [26], Tropicos database [27] and the International Plant Names Index [28]. Additional information was gathered from the study of numerous herbarium specimens, mainly from the Tropical Research Institute Herbarium (LISC, Lisbon, Portugal).

\section{Results and Discussion}

\section{Medicinal Plants' Survey}

A total of 53 plant species distributed over 47 genera and 31 families were reported by the 11 informants (Table 1). All the reported species grew naturally in the area, reflecting the social importance of the local floristic resources. Most of the identified plants were shrubs or trees (15 spp. or $28.3 \%$ ), herbs and trees (11 spp. for each category or $20.8 \%$ ), and shrubs (nine species or 17.0\%). The best represented families were Fabaceae (six species), Euphorbiaceae (four species) and Tiliaceae (three species). Altogether the 53 species were used to treat 50 different human health problems (Table 1), the great majority of which $(75.5 \%)$ having more than one medical application. The most cited species were Euclea racemosa (ca. 82\%), Colophospermum mopane, Cucumis sp. and Elephantorrhiza elephantina (ca. 73\% each species), Cassia abbreviata and Cissus quadrangularis (ca. 64\% each species), Aloe marlothii, Maerua edulis, Secamone parvifolia and Terminalia sericea (ca. 55\% each species) and Boscia albitrunca, Gossypium herbaceum and Gymnosporia heterophylla (ca. $46 \%$ each species) (data not shown). The number of medicinal plants and their potential applications reflect the rich ethnomedicinal knowledge in the Canhane community. Similar potentialities were found in other African countries like Cameroon [29] and Ethiopia [30-32] as well as in nonAfrican countries [33-35]. Certainly, there is a lot more knowledge to exploit on the topic in Mozambique.

More than half of the reported species (54.7\%) were used for stomach and intestine related disturbances (Table 2). Of these, almost 38\% were used to treat diarrhea and dysentery, a major concern in the region. In fact, in Mozambique diarrhea has for a long time been associated with a complex array of illnesses. Amongst them, dysentery and cholera usually have a high mortality rate if not treated promptly [10]. The use of traditional medicinal plants seems to play a major role in controlling diarrhea-associated diseases.

Around $23 \%$ of the surveyd species were used as analgesic, anti-inflammatory or anti-pyretic and for wound treatment, $15 \%$ for dentistry and $11 \%$ for gynecology-related problems. Approximately $9 \%$ of the reported species were used to treat ear diseases and hemorrhoids, $8 \%$ for burns, cough, debility and malnutrition, epilepsy, eye diseases and malaria, and 6\% for heart problems. Only one species, Ximenia americana (ca. 2\%) was used against HIV-AIDS. Thus, looking at the three major national health concerns, namely diarrhea and dysentery, malaria and HIV-AIDS a considerable number of potentialities are available for the first group (11 species), while moderate (four species) and low (one species) alternatives can be exploited for malaria and HIV-AIDS. In fact, several pharmacological studies of these three groups of human ailments are available for most of the species reported in the present survey [36-42].

With the exception of six species (Blepharis diversispina, Grewia flavescens, Guibourtia conjugata, Hermannia micropetala, Loeseneriella crenata, Zanthoxylum humile), all species under study have been reported as medicinal plants in other African countries [11,43-46]. While the use of G. flavescens and Z. humile by traditional healers has been reported in India and Mozambique, respectively [7,47], as far as our literature review goes, four species (i.e. B. diversispina, G. conjugata, $H$. micropetala, and L. crenata) were reported here for the first time. Of these, only two genera have been associated with ethonomedicine: the genus Blepharis [48] and the genus Loeseneriella (L. obtusifolia). Thus, these species constitute new potential sources of natural medicines.

From the 53 species, nine were reported previously by [10] and 3 by [7] in studies conducted in the province of Maputo. Besides that, several other species belonging to 11 genera (Aloe, Asparagus, Boscia, Cissus, Crinum, Cucumis, Ficus, Grewia, Maerua, Secamone, Strychnos) were also reported as medicinal species [7]. The potential medicinal plant markets from the southern provinces of Maputo and Gaza seem to be different. This may reflect the rich ethonomedicinal potential which exists in the entire country.

A comparative analysis with local specific ethnobotanical literature $[11,12,46]$ and complementary information gathered from the LISC Herbarium plant collections, identified 25 different plant species used for medicinal purposes (Table 3) of which only two, Combretum imberbe and Lannea schweinfurthii, are common to those reported in this study. Regarding their applications, similarities were found for $C$. imberbe (stomach 
Table 1 Medicinal plants (53 spp.) used in the Canhane village (2007- 2008)

\begin{tabular}{|c|c|c|c|c|c|c|}
\hline Scientific name* & $\begin{array}{l}\text { Local and Common } \\
\text { name }\end{array}$ & Habit & Part used & Main Diseases & $\begin{array}{l}\text { Administration } \\
\text { Route }\end{array}$ & $\begin{array}{l}\text { Method of } \\
\text { preparation }\end{array}$ \\
\hline \multicolumn{7}{|l|}{ Acanthaceae } \\
\hline \multirow{5}{*}{$\begin{array}{l}\text { Blepharis } \\
\text { diversispina (Nees) } \\
\text { C.B. Clarke }\end{array}$} & $\begin{array}{l}\text { Nchachacha wa mananga } \\
\text { Velvet bushwillow }\end{array}$ & $\begin{array}{l}\text { Sub-shrub } \\
\text { or Herb }\end{array}$ & Fruits & Hemorrhoids & Topic & $\begin{array}{l}\text { Burning and } \\
\text { grinding }\end{array}$ \\
\hline & & & & Cough & Oral & $\begin{array}{l}\text { Grinding and } \\
\text { maceration }\end{array}$ \\
\hline & & & Roots & Hemorrhoids & Topic & $\begin{array}{l}\text { Burning and } \\
\text { grinding }\end{array}$ \\
\hline & & & Seeds & Wounds & Topic & $\begin{array}{l}\text { Burning and } \\
\text { grinding }\end{array}$ \\
\hline & & & & Fontanel hardening & Topic & $\begin{array}{l}\text { Burning and } \\
\text { grinding }\end{array}$ \\
\hline \multicolumn{7}{|l|}{ Aloaceae } \\
\hline \multirow[t]{4}{*}{$\begin{array}{l}\text { Aloe marlothii A. } \\
\text { Berger }\end{array}$} & $\begin{array}{l}\text { Mhanga } \\
\text { Flat-flowered aloe, } \\
\text { Mountain aloe }\end{array}$ & Herb & $\begin{array}{l}\text { Leaves } \\
\text { (sap) }\end{array}$ & Biliary disorder; Malaria & Oral & Direct \\
\hline & & & & Wounds & Topic & Direct \\
\hline & & & $\begin{array}{l}\text { Roots; } \\
\text { Leaves }\end{array}$ & Toothache & Oral & Direct; Decoction \\
\hline & & & & Liver disorder & Oral & Decoction \\
\hline \multirow[t]{3}{*}{ Aloe zebrina Baker } & $\begin{array}{l}\text { Ximhangani } \\
\text { Small maculate aloe }\end{array}$ & Herb & $\begin{array}{l}\text { Leaves } \\
\text { (sap) }\end{array}$ & Eye treatments & Eyewash & Direct; Maceration \\
\hline & & & & Wounds & Topic & Direct \\
\hline & & & Roots & Liver disorder & Oral & Decoction \\
\hline \multicolumn{7}{|l|}{ Amaryllidaceae } \\
\hline $\begin{array}{l}\text { Crinum } \\
\text { stuhlmannii Baker }\end{array}$ & $\begin{array}{l}\text { Khonwua } \\
\text { Candy-striped crinum }\end{array}$ & Herb & Stem & Swellings & Topic & Decoction \\
\hline \multicolumn{7}{|l|}{ Anacardiaceae } \\
\hline \multirow[t]{2}{*}{$\begin{array}{l}\text { Lannea } \\
\text { schweinfurthii } \\
\text { (Engl.) Engl. }\end{array}$} & $\begin{array}{l}\text { Xivombo nkanyi, } \\
\text { xihumbunkany, } \\
\text { munganikomo } \\
\text { False marula }\end{array}$ & Tree & Bark & $\begin{array}{l}\text { Anemia; Diarrhea; Stomach } \\
\text { disorders }\end{array}$ & Oral & Decoction \\
\hline & & & Stem & Malaria & Oral & Decoction \\
\hline \multirow[t]{4}{*}{$\begin{array}{l}\text { Sclerocarya birrea } \\
\text { (A. Rich.) Hochst. }\end{array}$} & $\begin{array}{l}\text { Nkanyi } \\
\text { Marula }\end{array}$ & Tree & Bark & Anemia & Oral & Infusion \\
\hline & & & & Diarrhea; Stomach disorders & Oral & $\begin{array}{l}\text { Scraping and } \\
\text { decoction }\end{array}$ \\
\hline & & & & Hemorrhoids & Topic & $\begin{array}{l}\text { Decoction and } \\
\text { vapors; Scraping }\end{array}$ \\
\hline & & & Stem & Anemia & Oral & Maceration \\
\hline \multicolumn{7}{|l|}{ Apocynaceae } \\
\hline \multirow[t]{2}{*}{$\begin{array}{l}\text { Sarcostemma } \\
\text { viminale (L.) R. Br. }\end{array}$} & $\begin{array}{l}\text { Neta, netha } \\
\text { Caustic vine }\end{array}$ & $\begin{array}{l}\text { Herb } \\
\text { (succulent) }\end{array}$ & Root & Stomach ache & Oral & Decoction \\
\hline & & & Sap & Eye treatments & Eyewash & Direct \\
\hline \multirow{4}{*}{$\begin{array}{l}\text { Secamone } \\
\text { parvifolia (Oliv.) } \\
\text { Bullock }\end{array}$} & $\begin{array}{l}\text { Nyokani, nyoka ya yitsongo } \\
\text { Milimili }\end{array}$ & Shrub & Roots & Deworming; Rheumatism & Oral & Decoction \\
\hline & & & & Epilepsy & Oral & $\begin{array}{l}\text { Decoction; Heating; } \\
\text { Grinding and water }\end{array}$ \\
\hline & & & & Stomach ache & Oral & $\begin{array}{l}\text { Crushing and water; } \\
\text { Decoction }\end{array}$ \\
\hline & & & $\begin{array}{l}\text { Stem; } \\
\text { Roots }\end{array}$ & Varicose veins & Topic & Decoction \\
\hline
\end{tabular}


Table 1: Medicinal plants (53 spp.) used in the Canhane village (2007- 2008) (Continued)

\begin{tabular}{|c|c|c|c|c|c|c|}
\hline \multicolumn{7}{|l|}{ Asparagaceae } \\
\hline \multirow[t]{2}{*}{$\begin{array}{l}\text { Asparagus } \\
\text { africanus Lam. }\end{array}$} & $\begin{array}{l}\text { Kwangwa la tilo } \\
\text { Bush asparagus }\end{array}$ & Shrub & Roots & Stomach disorders & Oral & Decoction \\
\hline & & & $\begin{array}{l}\text { Whole } \\
\text { plant }\end{array}$ & Stomach disorders & Oral & $\begin{array}{l}\text { Grinding and } \\
\text { maceration }\end{array}$ \\
\hline \multicolumn{7}{|l|}{ Balanitaceae } \\
\hline $\begin{array}{l}\text { Balanites } \\
\text { maughamii } \\
\text { Sprague }\end{array}$ & $\begin{array}{l}\text { Nulu, nulo } \\
\text { Green thorn, Y-thorned }\end{array}$ & Tree & Roots & Malaria & Oral & $\begin{array}{l}\text { Scraping and } \\
\text { infusion }\end{array}$ \\
\hline \multicolumn{7}{|l|}{ Bombacaceae } \\
\hline \multirow[t]{2}{*}{$\begin{array}{l}\text { Adansonia digitata } \\
\mathrm{L} \text {. }\end{array}$} & $\begin{array}{l}\text { Ximuwa, ximuhu, ximuvo } \\
\text { Baobab }\end{array}$ & Tree & Bark & Debility & Bath & Maceration \\
\hline & & & Roots & Diarrhea & Oral & Maceration \\
\hline \multicolumn{7}{|l|}{ Capparaceae } \\
\hline $\begin{array}{l}\text { Boscia albitrunca } \\
\text { (Burch.) Gilg \& } \\
\text { Gilg-Ben. }\end{array}$ & $\begin{array}{l}\text { Nxunkutso, xikutse, xikutso, } \\
\text { xikutsu, xukutsi } \\
\text { Shepherd's tree }\end{array}$ & $\begin{array}{l}\text { Shrub or } \\
\text { tree }\end{array}$ & Leaves & Diarrhea; Hemorrhoids & Topic & $\begin{array}{l}\text { Crushing and } \\
\text { infusion }\end{array}$ \\
\hline $\begin{array}{l}\text { Boscia foetida } \\
\text { Schinz subsp. } \\
\text { filipes (Gilg) Lötter }\end{array}$ & $\begin{array}{l}\text { Xicutso } \\
\text { Bushveld shepherds tree, } \\
\text { sandveld shepherds, tree } \\
\text { smelly shepherds tree }\end{array}$ & Shrub & Roots & Stomach and kidney purification & Oral & Infusion \\
\hline \multirow{2}{*}{$\begin{array}{l}\text { Maerua edulis } \\
\text { (Gilg \& Gilg-Ben. ) } \\
\text { De Wolf }\end{array}$} & $\begin{array}{l}\text { Xikolwa, xikolwe } \\
\text { Blue-leaved bush cherry }\end{array}$ & $\begin{array}{l}\text { Suffrutex } \\
\text { or Shrub }\end{array}$ & Roots & Women fertility, & Oral & Decoction; Infusion \\
\hline & & & & Stomach ache & Oral & Decoction \\
\hline $\begin{array}{l}\text { Maerua parvifolia } \\
\text { Pax }\end{array}$ & $\begin{array}{l}\text { Nongonoko } \\
\text { Dwarf bush-cherry, small- } \\
\text { leaved maerua }\end{array}$ & Shrub & Roots & $\begin{array}{l}\text { Diarrhea; Stomach ache and } \\
\text { purification }\end{array}$ & Oral & Decoction \\
\hline \multicolumn{7}{|l|}{ Celastraceae } \\
\hline \multirow{3}{*}{$\begin{array}{l}\text { Loeseneriella } \\
\text { crenata (Klotzsch) } \\
\text { Wilczek ex N.Hallé }\end{array}$} & $\begin{array}{l}\text { Lorho, nhlohlo } \\
\text { Valley paddle-pod }\end{array}$ & $\begin{array}{l}\text { Climbing } \\
\text { shrub }\end{array}$ & Roots & Epilepsy; Stomach ache & Oral & Decoction \\
\hline & & & & Malnutrition & Oral; Vaccine & $\begin{array}{l}\text { Burning and } \\
\text { decoction; Grinding; } \\
\text { Scraping and } \\
\text { burning }\end{array}$ \\
\hline & & & Stem & Antialergic & Necklace & Direct \\
\hline \multirow[t]{3}{*}{$\begin{array}{l}\text { Gymnosporia } \\
\text { heterophylla (Eckl. } \\
\text { \& Zeyh.) Loes. }\end{array}$} & $\begin{array}{l}\text { Xivambulani, xichangwa, } \\
\text { libatzondze } \\
\text { Angular-stemmed } \\
\text { spikethorn, common } \\
\text { spikethorn }\end{array}$ & $\begin{array}{l}\text { Shrub or } \\
\text { Small tree }\end{array}$ & Leaves & Swellings & Oral & Decoction \\
\hline & & & Roots & Internal clots & Oral & Decoction \\
\hline & & & & Stabbing heart & Oral; Topic & $\begin{array}{l}\text { Burning and } \\
\text { grinding; Decoction; } \\
\text { Scraping }\end{array}$ \\
\hline \multicolumn{7}{|l|}{ Combretaceae } \\
\hline \multirow[t]{2}{*}{$\begin{array}{l}\text { Combretum } \\
\text { imberbe Wawra }\end{array}$} & $\begin{array}{l}\text { Mondzo } \\
\text { Leadwood }\end{array}$ & $\begin{array}{l}\text { Shrub or } \\
\text { tree }\end{array}$ & Bark & Toothache & Oral & Decoction \\
\hline & & & NA & Stomach ache & Oral & $\begin{array}{l}\text { Burning and } \\
\text { watering }\end{array}$ \\
\hline \multirow[t]{3}{*}{$\begin{array}{l}\text { Terminalia sericea } \\
\text { Burch. ex DC. }\end{array}$} & $\begin{array}{l}\text { Nsunsu, nkonola, kondla, } \\
\text { mogonono } \\
\text { Silver cluster-leaf, silver } \\
\text { terminalia }\end{array}$ & Tree & $\begin{array}{l}\text { Branches } \\
\text { (bark) }\end{array}$ & Burns; Wounds & Topic & Drying and grinding \\
\hline & & & Leaves & Stomach ache & Oral & Decoction \\
\hline & & & Roots & Diarrhea & Oral & Decoction \\
\hline
\end{tabular}


Table 1: Medicinal plants (53 spp.) used in the Canhane village (2007- 2008) (Continued)

\begin{tabular}{|c|c|c|c|c|c|c|}
\hline & & & & Burns; Wounds & Topic & $\begin{array}{l}\text { Drying and } \\
\text { grinding; Scraping }\end{array}$ \\
\hline \multicolumn{7}{|l|}{ Cucurbitaceae } \\
\hline $\begin{array}{l}\text { Cucumis } \\
\text { metuliferus E.Mey. } \\
\text { ex Naudin }\end{array}$ & Dema & Herb & Roots & Appendicitis; Stomach ache & Oral & Decoction \\
\hline \multirow[t]{3}{*}{$\begin{array}{l}\text { Cucumis zeyheri } \\
\text { Sond. }\end{array}$} & $\begin{array}{l}\text { Xiyakayani, xihakahani, } \\
\text { chihacaiane } \\
\text { Wild cucumber }\end{array}$ & Herb & Fruits & Stomach disorders & Oral & Drying and grinding \\
\hline & & & & Laxative & Enema & $\begin{array}{l}\text { Decanting and } \\
\text { filtration; Grinding; } \\
\text { Maceration }\end{array}$ \\
\hline & & & Leaves & Dysentery; Laxative & Oral & $\begin{array}{l}\text { Decoction; } \\
\text { Maceration }\end{array}$ \\
\hline \multicolumn{7}{|l|}{ Dracaenaceae } \\
\hline \multirow{3}{*}{$\begin{array}{l}\text { Sansevieria } \\
\text { hyacinthoides (L.) } \\
\text { Druce }\end{array}$} & $\begin{array}{l}\text { Xikwenga xa kwhati } \\
\text { Mother-in-law tongue }\end{array}$ & Herb & Leaves & $\begin{array}{l}\text { Contusions; Hemorrhoids; } \\
\text { Rheumatism; Swellings; }\end{array}$ & Topic & $\begin{array}{l}\text { Decoction and } \\
\text { vapors; Heating }\end{array}$ \\
\hline & & & Roots & Women fertility & Oral & Crushing and water \\
\hline & & & & Epilepsy & Oral & Decoction \\
\hline \multicolumn{7}{|l|}{ Ebenaceae } \\
\hline \multirow[t]{3}{*}{$\begin{array}{l}\text { Euclea racemosa } \\
\text { Murr. }\end{array}$} & $\begin{array}{l}\text { Mulala, nhlangulo } \\
\text { Bush guarri, glossy guarri } \\
\text { river guarri }\end{array}$ & Shrub & Roots & Caries; Toothache & Oral & Direct (chewing) \\
\hline & & & & Wounds & Topic & $\begin{array}{l}\text { Peeling and } \\
\text { grinding }\end{array}$ \\
\hline & & & Stem & Wounds & Bath & Cutting and water \\
\hline \multicolumn{7}{|l|}{ Euphorbiaceae } \\
\hline \multirow[t]{5}{*}{ Acalypha indica $\mathrm{L}$. } & $\begin{array}{l}\text { Ntlambissana } \\
\text { Copperleaf, indian nettle }\end{array}$ & Herb & Leaves & Hemorrhoids & Oral; Topic & Crushing; Decoction \\
\hline & & & & Intestinal lavage & Enema & $\begin{array}{l}\text { Grinding and } \\
\text { decoction; } \\
\text { Maceration }\end{array}$ \\
\hline & & & & Laxative & Oral; Topic & Crushing; Infusion \\
\hline & & & Roots & Laxative & Oral & Decoction; Infusion \\
\hline & & & Stem & Hemorrhoids & Oral & Decoction \\
\hline $\begin{array}{l}\text { Androstachys } \\
\text { johnsonii Prain }\end{array}$ & $\begin{array}{l}\text { Cimbiri } \\
\text { Lebombo-ironwood, simbi } \\
\text { tree }\end{array}$ & Tree & NA & NA & NA & $\begin{array}{l}\text { Kept in secret by } \\
\text { Hitch doctors }\end{array}$ \\
\hline $\begin{array}{l}\text { Flueggea virosa } \\
\text { (Roxb. ex Willd.) } \\
\text { Voigt }\end{array}$ & $\begin{array}{l}\text { Nsangasi, sangasi } \\
\text { Snowberry tree, whiteberry } \\
\text { bush }\end{array}$ & Shrub & Branches & Abcesses & Topic & $\begin{array}{l}\text { Heating (with castor } \\
\text { oil on top) }\end{array}$ \\
\hline \multirow[t]{3}{*}{$\begin{array}{l}\text { Spirostachys } \\
\text { africana Sond. }\end{array}$} & $\begin{array}{l}\text { Xilangamahlo, dzanvori } \\
\text { African Sandal, tamboti }\end{array}$ & Tree & Bark & Debility (HIV-AIDS) & Oral & Decoction (in milk) \\
\hline & & & Sap & Ear and eye treatments & Topic & Direct \\
\hline & & & Stem & Burns, Wounds & Topic & $\begin{array}{l}\text { Burning; Grinding; } \\
\text { Scraping }\end{array}$ \\
\hline \multicolumn{7}{|l|}{ Fabaceae } \\
\hline \multirow[t]{4}{*}{$\begin{array}{l}\text { Cassia abbreviata } \\
\text { Oliv. }\end{array}$} & $\begin{array}{l}\text { Lumanyama } \\
\text { Longtail cassia, sjambok } \\
\text { pod }\end{array}$ & Tree & Fruits & Eye treatments & Eyewash & $\begin{array}{l}\text { Heating and } \\
\text { grinding }\end{array}$ \\
\hline & & & $\begin{array}{l}\text { Leaves, } \\
\text { roots and } \\
\text { stems } \\
\text { (mix) }\end{array}$ & Stomach ache & Oral & Infusion \\
\hline & & & $\begin{array}{l}\text { Roots } \\
\text { (bark) }\end{array}$ & Diarrhea & Oral & Decoction \\
\hline & & & Stem & Malaria; Stomach ache & Oral & Decoction \\
\hline
\end{tabular}


Table 1: Medicinal plants (53 spp.) used in the Canhane village (2007- 2008) (Continued)

\begin{tabular}{|c|c|c|c|c|c|c|}
\hline \multirow[t]{5}{*}{$\begin{array}{l}\text { Colophospermum } \\
\text { mopane (Benth.) } \\
\text { Léonard }\end{array}$} & \multirow[t]{5}{*}{$\begin{array}{l}\text { Gungwa, nxanati, nxanatsi, } \\
\text { mesanya } \\
\text { Mopane }\end{array}$} & \multirow[t]{5}{*}{$\begin{array}{l}\text { Shrub or } \\
\text { Tree }\end{array}$} & Bark & $\begin{array}{l}\text { Bleeding; Dysentery; Stomach } \\
\text { ache }\end{array}$ & Oral & Decoction \\
\hline & & & \multirow[t]{2}{*}{ Leaves } & Stomach ache & Oral & $\begin{array}{l}\text { Crushing; Direct } \\
\text { (chewing); Infusion }\end{array}$ \\
\hline & & & & Dysentery & Oral & Grinding and water \\
\hline & & & Roots & Stomach ache & Oral & Decoction \\
\hline & & & $\begin{array}{l}\text { Stem; } \\
\text { Stem and } \\
\text { leaves } \\
\text { (mix) }\end{array}$ & Stomach ache; Diarrhea & Oral & Decoction; Infusion \\
\hline $\begin{array}{l}\text { Dalbergia } \\
\text { melanoxylon Guill. } \\
\& \text { Perr. }\end{array}$ & $\begin{array}{l}\text { Xipaladze, xiphalanzi } \\
\text { African blackwood }\end{array}$ & $\begin{array}{l}\text { Shrub or } \\
\text { Tree }\end{array}$ & Roots & Toothache & Oral & Decoction \\
\hline \multirow{2}{*}{$\begin{array}{l}\text { Dichrostachys } \\
\text { cinerea (L.) Wight } \\
\& \text { Arn }\end{array}$} & \multirow{2}{*}{$\begin{array}{l}\text { Ndzenga, ntsenga, } \\
\text { ndzhenga } \\
\text { Small-leaved sickle bush }\end{array}$} & \multirow[t]{2}{*}{$\begin{array}{l}\text { Shrub or } \\
\text { Small tree }\end{array}$} & Roots & Skeletal disorders & Topic; Vaccine & $\begin{array}{l}\text { Burning and } \\
\text { grinding; Scraping }\end{array}$ \\
\hline & & & $\begin{array}{l}\text { Roots } \\
\text { (sap) }\end{array}$ & Laxative & Oral & Direct \\
\hline \multirow[t]{2}{*}{$\begin{array}{l}\text { Elephantorrhiza } \\
\text { elephantina } \\
\text { (Burch.) Skeels }\end{array}$} & \multirow[t]{2}{*}{$\begin{array}{l}\text { Xivurayi } \\
\text { Dwarf elephant's root }\end{array}$} & \multirow[t]{2}{*}{$\begin{array}{l}\text { Shrub or } \\
\text { Sub-shrub }\end{array}$} & \multirow[t]{2}{*}{ Roots } & Anemia & Oral & $\begin{array}{l}\text { Cutting and } \\
\text { maceration; } \\
\text { Decoction and } \\
\text { grinding }\end{array}$ \\
\hline & & & & Pain killer, Fever & Oral & Decoction \\
\hline \multirow{2}{*}{$\begin{array}{l}\text { Guibourtia } \\
\text { conjugata (Bolle) } \\
\text { J. Léonard }\end{array}$} & \multirow{2}{*}{$\begin{array}{l}\text { Ntsotso } \\
\text { Small copalwood, small } \\
\text { false mopane }\end{array}$} & \multirow[t]{2}{*}{ Tree } & Leaves & Stomach disorders & Oral & Crushing and water \\
\hline & & & Roots & Intense cough & Oral & Decoction \\
\hline \multicolumn{7}{|l|}{ Malvaceae } \\
\hline \multirow[t]{3}{*}{$\begin{array}{l}\text { Gossypium } \\
\text { herbaceum L. }\end{array}$} & \multirow[t]{3}{*}{$\begin{array}{l}\text { Thonji ra khwati, thondji la } \\
\text { khwati, nuba } \\
\text { Wild cotton }\end{array}$} & \multirow[t]{3}{*}{ Sub-shrub } & Fruits & Ear treatment & Topic & Direct \\
\hline & & & \multirow[t]{2}{*}{ Roots } & Vomits control & Oral & Decoction \\
\hline & & & & Tonic & Oral & Decoction \\
\hline $\begin{array}{l}\text { Hibiscus meyeri } \\
\text { Harv. }\end{array}$ & $\begin{array}{l}\text { Muxaxayevu, kongowa, } \\
\text { kloklonya, muchachanyevo } \\
\text { Dainty white wild hibiscus, } \\
\text { lebombo hibiscus }\end{array}$ & Herb & Roots & Tonic, Stabbing heart & Oral & Decoction \\
\hline \multicolumn{7}{|l|}{ Meliaceae } \\
\hline \multirow{2}{*}{$\begin{array}{l}\text { Trichilia emetica } \\
\text { Vahl subsp. } \\
\text { emetica }\end{array}$} & \multirow[t]{2}{*}{$\begin{array}{l}\text { Nkuhlu } \\
\text { Natal-mahogany }\end{array}$} & \multirow[t]{2}{*}{ Tree } & $\begin{array}{l}\text { Branches } \\
\text { (sap) }\end{array}$ & Stomach ache & Oral & Direct (chewing) \\
\hline & & & Roots & Contraceptive & Oral & Infusion \\
\hline \multicolumn{7}{|l|}{ Menispermaceae } \\
\hline \multirow[t]{3}{*}{$\begin{array}{l}\text { Tinospora caffra } \\
\text { (Miers) Troupin }\end{array}$} & \multirow[t]{3}{*}{$\begin{array}{l}\text { Nyokani ya yikulo, nyoka ya } \\
\text { yikulu } \\
\text { Orange grape creeper }\end{array}$} & \multirow[t]{3}{*}{ Creeper } & Leaves & Paralysis and Children diseases & Oral & Decoction \\
\hline & & & Roots & $\begin{array}{l}\text { Epilepsy; Pain killer;"; Paralysis and } \\
\text { Children diseases; Stomach ache }\end{array}$ & Oral & Decoction; Infusion \\
\hline & & & Stem & Epilepsy & Oral & Decoction \\
\hline \multicolumn{7}{|l|}{ Moraceae } \\
\hline Ficus sycomorus L. & $\begin{array}{l}\text { Nkuwa } \\
\text { Common cluster fig, } \\
\text { sycamore fig }\end{array}$ & Tree & Sap & Ringworm & Topic & Direct \\
\hline \multicolumn{7}{|l|}{ Olacaceae } \\
\hline $\begin{array}{l}\text { Olax dissitiflora } \\
\text { Oliv. }\end{array}$ & $\begin{array}{l}\text { Nkondzomhuntana, } \\
\text { ximanimurhi, } \\
\text { nondzomuntana } \\
\text { Small sourplum, small-fruit } \\
\text { olax }\end{array}$ & $\begin{array}{l}\text { Shrub or } \\
\text { Tree }\end{array}$ & Leaves & Wounds & Topic & Grinding \\
\hline
\end{tabular}


Table 1: Medicinal plants (53 spp.) used in the Canhane village (2007- 2008) (Continued)

\begin{tabular}{|c|c|c|c|c|c|c|}
\hline \multirow[t]{2}{*}{$\begin{array}{l}\text { Ximenia } \\
\text { americana L. }\end{array}$} & \multirow[t]{2}{*}{$\begin{array}{l}\text { Ntsengele, matsengele, } \\
\text { tsingela } \\
\text { Blue sourplum, small } \\
\text { sourplum }\end{array}$} & \multirow[t]{2}{*}{$\begin{array}{l}\text { Shrub or } \\
\text { Tree }\end{array}$} & \multirow[t]{2}{*}{ Roots } & \multirow{2}{*}{$\begin{array}{l}\text { Antiabortifacients, HIV-AIDS, } \\
\text { Menstrual cycle, Stabbing heart, } \\
\text { Stomach ache, Women fertility, } \\
\text { Wounds }\end{array}$} & Oral & \multirow[b]{2}{*}{ Drying and grinding } \\
\hline & & & & & Topic & \\
\hline \multicolumn{7}{|l|}{ Orchidaceae } \\
\hline \multirow[t]{2}{*}{$\begin{array}{l}\text { Ansellia africana } \\
\text { Lindl. }\end{array}$} & $\begin{array}{l}\text { Phakama } \\
\text { Leopard orchid, monkey } \\
\text { sugarcane, mopane orchid, } \\
\text { tree orchid }\end{array}$ & $\begin{array}{l}\text { Herb } \\
\text { (Epiphyte) }\end{array}$ & Fruits & Cough, Rheumatism & Necklace, Bath & $\begin{array}{l}\text { Direct; Heating and } \\
\text { grinding }\end{array}$ \\
\hline & & & $\begin{array}{l}\text { Fruits and } \\
\text { stem (mix) }\end{array}$ & Cough & Oral & Decoction \\
\hline \multicolumn{7}{|l|}{ Poaceae } \\
\hline $\begin{array}{l}\text { Cynodon dactylon } \\
\text { (L.) Pers. }\end{array}$ & $\begin{array}{l}\text { Rintlhangi, nulangi-rithangi } \\
\text { Bermuda grass }\end{array}$ & Herb & Leaves & Antiabortifacients & Oral & Grinding and water \\
\hline \multicolumn{7}{|l|}{ Ptaeroxylaceae } \\
\hline $\begin{array}{l}\text { Ptaeroxylon } \\
\text { obliquum Radlk. }\end{array}$ & $\begin{array}{l}\text { Ndzharhi } \\
\text { Sneezewood }\end{array}$ & $\begin{array}{l}\text { Shrub or } \\
\text { Tree }\end{array}$ & Sap & Stomach ache & Oral & Direct \\
\hline \multicolumn{7}{|l|}{ Rubiaceae } \\
\hline $\begin{array}{l}\text { Gardenia volkensii } \\
\text { K. Schum. }\end{array}$ & $\begin{array}{l}\text { Xitsalala } \\
\text { Bushveld, savanna or } \\
\text { woodland gardenia, } \\
\text { transvaal gardenia }\end{array}$ & $\begin{array}{l}\text { Shrub or } \\
\text { Tree }\end{array}$ & Leaves & Stomach ache & Oral & Grinding and water \\
\hline \multicolumn{7}{|l|}{ Rutaceae } \\
\hline \multirow[t]{3}{*}{$\begin{array}{l}\text { Zanthoxylum } \\
\text { humile (E.A. Bruce) } \\
\text { P. G. Waterman }\end{array}$} & $\begin{array}{l}\text { Manungwani, } \\
\text { manongwane, } \\
\text { manungwame } \\
\text { Hairy knobwood }\end{array}$ & Shrub & Roots & Mouth anesthetic; Toothache & Oral; Topic & $\begin{array}{l}\text { Decoction; Peeling, } \\
\text { grinding, drying. } \\
\text { and grinding }\end{array}$ \\
\hline & & & & Wounds and Burns & Topic & $\begin{array}{l}\text { Peeling, grinding, } \\
\text { drying. and } \\
\text { grinding }\end{array}$ \\
\hline & & & Stem & Pain killer & Vaccine & Burning \\
\hline \multicolumn{7}{|l|}{ Sapotaceae } \\
\hline \multirow[t]{2}{*}{$\begin{array}{l}\text { Manilkara } \\
\text { mochisia (Baker) } \\
\text { Dubard }\end{array}$} & $\begin{array}{l}\text { N'whamba, wambo, } \\
\text { n'wambu } \\
\text { Lowveld milkberry }\end{array}$ & $\begin{array}{l}\text { Shrub or } \\
\text { tree }\end{array}$ & Roots & Toothache & Oral; Topic & $\begin{array}{l}\text { Decoction; } \\
\text { Maceration and } \\
\text { scrapping }\end{array}$ \\
\hline & & & & Ear treatments & Topic & Direct \\
\hline \multicolumn{7}{|l|}{ Strychnaceae } \\
\hline $\begin{array}{l}\text { Strychnos } \\
\text { madagascariensis } \\
\text { Spreng. ex Baker }\end{array}$ & $\begin{array}{l}\text { Nkwankwa } \\
\text { Black monkey-orange, } \\
\text { hairy-leaved monkey- } \\
\text { orange }\end{array}$ & $\begin{array}{l}\text { Shrub or } \\
\text { Tree }\end{array}$ & Roots & Fever & Oral & $\begin{array}{l}\text { Peeling and } \\
\text { decoction }\end{array}$ \\
\hline \multicolumn{7}{|l|}{ Sterculiaceae } \\
\hline \multirow{3}{*}{$\begin{array}{l}\text { Hermannia } \\
\text { micropetala Harv. } \\
\text { \& Sond. }\end{array}$} & $\begin{array}{l}\text { Sindzambita, xisindzambita } \\
\text { Cactus wine, wild grape }\end{array}$ & $\begin{array}{l}\text { Shrub or } \\
\text { Sub-shrub }\end{array}$ & Fruits & Laxative & Topic & Juice \\
\hline & & & Leaves & Laxative & Topic & Juice \\
\hline & & & Roots & Fontanel hardening & Topic & Burning and oil \\
\hline \multicolumn{7}{|l|}{ Tiliaceae } \\
\hline $\begin{array}{l}\text { Grewia flavescens } \\
\text { Juss. var. } \\
\text { flavescens }\end{array}$ & $\begin{array}{l}\text { Nsihana, nsiphane, dzuwa } \\
\text { wa mananga } \\
\text { Donkeyberry, Sandpaper } \\
\text { raisin, Rough-leaved raisin }\end{array}$ & $\begin{array}{l}\text { Climbing } \\
\text { shrub }\end{array}$ & Leaves & Stomach disorders & NA & NA \\
\hline \multirow[t]{2}{*}{$\begin{array}{l}\text { Grewia hexamita } \\
\text { Burret }\end{array}$} & $\begin{array}{l}\text { Nsihana, nsihani, nsihane, } \\
\text { nsiphane } \\
\text { Giant grewia, Giant raisin }\end{array}$ & $\begin{array}{l}\text { Shrub or } \\
\text { Tree }\end{array}$ & Roots & Menstrual cycle, Women Fertility & Oral & Infusion \\
\hline & & & Sap & Post-delivery cleaning & Oral & Direct \\
\hline
\end{tabular}


Table 1: Medicinal plants (53 spp.) used in the Canhane village (2007- 2008) (Continued)

\begin{tabular}{|c|c|c|c|c|c|c|}
\hline \multirow[t]{4}{*}{$\begin{array}{l}\text { Grewia monticola } \\
\text { Sond. }\end{array}$} & \multirow[t]{4}{*}{$\begin{array}{l}\text { Nsihana, nsihani, nsiphane } \\
\text { Grey grewia, Grey raisin, } \\
\text { Silver raisin }\end{array}$} & \multirow[t]{4}{*}{$\begin{array}{l}\text { Shrub or } \\
\text { Tree }\end{array}$} & Fruits & Ear treatments & Topic & $\begin{array}{l}\text { Heating and } \\
\text { grinding }\end{array}$ \\
\hline & & & $\begin{array}{l}\text { Fruits; } \\
\text { Seeds }\end{array}$ & Wounds & Topic & $\begin{array}{l}\text { Heating and } \\
\text { grinding }\end{array}$ \\
\hline & & & Roots & Diarrhea & Oral & Decoction \\
\hline & & & Stem & Swellings & Topic & Heating \\
\hline \multicolumn{7}{|l|}{ Vitaceae } \\
\hline $\begin{array}{l}\text { Cissus cornifolia } \\
\text { (Bak.) Planch. }\end{array}$ & $\begin{array}{l}\text { Mphesani, mphensana } \\
\text { Ivy-grape }\end{array}$ & $\begin{array}{l}\text { Shrub or } \\
\text { Tree }\end{array}$ & Roots & Burns; Wounds & Topic & Crushing \\
\hline \multirow[t]{6}{*}{$\begin{array}{l}\text { Cissus } \\
\text { quadrangularis } \mathrm{L} \text {. }\end{array}$} & \multirow[t]{6}{*}{$\begin{array}{l}\text { Covoloti, Covoluti } \\
\text { Cactus vine, wild grape }\end{array}$} & \multirow[t]{6}{*}{$\begin{array}{l}\text { Creeper } \\
\text { (succulent) }\end{array}$} & Roots & Ear treatments & Topic & $\begin{array}{l}\text { Warming and } \\
\text { squeezing }\end{array}$ \\
\hline & & & Sap & Ear treatments; Wounds & Topic & Direct \\
\hline & & & Seeds & Antidote; Wounds & Topic & Grinding \\
\hline & & & \multirow[t]{3}{*}{ Stem } & Sprains; Swellings & Topic & Heating \\
\hline & & & & Cough & Oral & $\begin{array}{l}\text { Cutting and } \\
\text { decoction }\end{array}$ \\
\hline & & & & Antidote; Wounds & Topic & Crushing and water \\
\hline
\end{tabular}

Family, scientific, local and common names, growth habit, parts used, main diseases, administration route and method of preparation.

*Scientific names are according to [22-28].

$\mathrm{NA}=$ Not Available.

disorders) and L. schweinfurthii (diarrhea and stomach disorders). According to the available data, C. imberbe was also used to treat schistosomiasis and L. schweinfurthii to treat tuberculosis, while in our survey they were additionally indicated for the treatment of toothache (C. imberbe), anemia and malaria (L. schweinfurthii). Because the older surveys did not specifically target medicinal plants, we believe that our data are more accurate in what concerns the applications of these two species. This fact may also explain why the great majority of the species reported 40 years ago (23 out of 25 or $92 \%$ ) does not overlap with those identified in this survey. However, the possibility of loss of genetic resources and/or traditional knowledge should also be considered.

The great majority of the identified species (46 spp. or $86.8 \%$ ) were also used for other purposes than medicine (Table 4; Figure 2). The major groups of applications were associated with beliefs and myths (26 spp. or ca. $49 \%$ ) or used as food (24 spp. or ca. $45 \%$ ). Wood production, handicraft and veterinary were the third major class of application, with 10 (ca. 19\%), 9 (ca. 17\%) and 8 (ca. 15\%) species, respectively. This reinforces the socioeconomic importance of the reported species, placing them in a privileged position for conservational aspects and income-generating purposes.

\section{Plant parts used, methods of preparation and administration routes}

Several plant parts were used (Table 1), the most frequent being roots $(38.8 \%)$, followed by leaves $(17.5 \%)$, stems (13.6\%), fruits (8.8\%), bark (5.8\%), sap (5.8\%), combinations of plant organs (3.9\%), branches $(2.9 \%)$ and seeds $(2.9 \%)$. Regarding the methods of preparation (Figure 3), in many cases (38\%) a combination of methods was used. The most common method was decoction (25\%), followed by direct consumption (10\%), infusion $(6 \%)$, crushing $(5 \%)$, grinding $(5 \%)$, maceration $(4 \%)$, scraping (2\%), heating (2\%), burning (1\%), cutting (1\%) and juice $(1 \%)$. Fifty nine percent of the medicines were administered orally, $31 \%$ topically and only $10 \%$ through vaccine, bath, enema, eyewash and necklace (ca. $2 \%$ for each mode) (Figure 4). In general, the results seem to follow the pattern of medicinal plant uses in Africa $[26,28,49]$ except that in Canhane, instead of leaves, roots occupy the top position which is concordant with the results from [7]. Consistent with the findings of $[28,49]$ in Kenya and Ethiopia respectively, is the lack of standardized dosage and quality control.

\section{Conservational aspects}

In general, the community was conscientious and motivated regarding conservational issues and had adopted sound measures for the rational use of medicinal plants. Conservation in farms or home gardens was performed for the most commonly used plants, namely Aloe marlothii, A. zebrina, B. albitrunca, C. mopane, C. zeyheri, E. racemosa, Ficus sycomorus, Flueggea virosa, Grewia hexamita, G. monticola, H. micropetala, Sclerocarya birrea and T. sericea. Additionally, the intensity and frequency of exploitation was controlled and there were 


\section{Table 2 Distribution by category of disease}

\begin{tabular}{|c|c|}
\hline $\begin{array}{l}\text { Analgesic, anti-inflammatory and } \\
\text { antipyretic }\end{array}$ & $\begin{array}{l}\text { Adansonia digitata, Cissus quadrangularis, Crinum stuhlmannii, Dichrostachys cinerea, Elephantorrhiza elephantina, } \\
\text { Grewia monticola, Gymnosporia heterophylla, Sansevieria hyacinthoides, Strychnos madagascariensis, Tinospora } \\
\text { caffra, Zanthoxylum humile }\end{array}$ \\
\hline Anemia & Elephantorrhiza elephantina, Lannea schweinfurthii, Sclerocarya birrea \\
\hline Antialergic & Loeseneriella crenata \\
\hline Antidote & Cissus quadrangularis \\
\hline Appendicitis & Cucumis metuliflerus \\
\hline Bleeding & Colophospermum mopane \\
\hline Burns & Cissus cornifolia, Spirostachys africana, Terminalia sericea, Zanthoxylum humile \\
\hline Cough & Ansellia africana, Blepharis diversispina, Cissus quadrangularis, Guibourtia conjugata \\
\hline Debility and malnutrition & Gossypium herbaceum, Hibiscus meyeri, Loeseneriella crenata, Spirostachys africana \\
\hline Dentistry & $\begin{array}{l}\text { Aloe marlothii, Boscia albitrunca, Combretum imberbe, Dalbergia melanoxylon, Euclea racemosa, Flueggea virosa, } \\
\text { Manilkara mochisia, Zanthoxylum humile }\end{array}$ \\
\hline Deworming & Secamone parvifolia \\
\hline Ear diseases & Cissus quadrangularis, Gossypium herbaceum, Grewia monticola, Manilkara mochisia, Spirostachys africana \\
\hline Epilepsy & Loeseneriella crenata, Sansevieria hyacinthoides, Secamone parvifolia, Tinospora caffra \\
\hline Eye diseases & Aloe zebrina, Cassia abbreviata, Sarcostemma viminale, Spirostachys africana \\
\hline Ginecology & Cynodon dactylon, Grewia hexamita, Maerua edulis, Sansevieria hyacinthoides, Trichilia emetica, Ximenia americana \\
\hline Heart & Gymnosporia heterophylla, Hibiscus meyeri, Ximenia americana \\
\hline Hemorrhoids & Acalypha indica, Blepharis diversispina, Boscia albitrunca, Sansevieria hyacinthoides, Sclerocarya birrea \\
\hline HIV-SIDA & Ximenia americana \\
\hline Internal clots & Gymnosporia heterophylla \\
\hline Kidney disorders & Boscia foetida \\
\hline Liver disorders & Aloe marlothii, Aloe zebrina \\
\hline Malaria & Aloe marlothii, Balanites maughamii, Cassia abbreviata, Lannea schweinfurthii \\
\hline Non-identified diseases & Androstachys johnsonii \\
\hline Paralysis and other children & Tinospora caffra \\
\hline
\end{tabular}
diseases

\begin{tabular}{ll}
\hline Ringworm & Ficus sycomorus \\
\hline Skeletal structure & Dichrostachys cinerea \\
\hline Stomach and intestine disorders & Acalypha indica, Adansonia digitata, Asparagus africanus, Boscia albitrunca, Boscia foetida, Cassia abbreviata, \\
& Colophospermum mopane, Combretum imberbe, Cucumis metuliflerus, Cucumis zeyheri, Dichrostachys cinerea, \\
& Hermannia micropetala, Gardenia volkensii, Grewia flavescens, Grewia monticola, Guibourtia conjugata, Gossypium \\
& herbaceum, Lannea schweinfurthii, Loeseneriella crenata, Maerua edulis, Maerua parvifolia, Ptaeroxylon obliquum, \\
& Sarcostemma viminale, Secamone parvifolia, Sclerocarya birrea, Terminalia sericea, Tinospora caffra, Trichilia emetica, \\
& Ximenia americana \\
\hline Varicose veins & Secamone parvifolia \\
\hline Wounds & Aloe marlothii, Aloe zebrina, Blepharis diversispina, Cissus cornifolia, Cissus quadrangularis, Euclea racemosa, Grewia \\
& monticola, Olax dissitiflora, Spirostachys africana, Terminalia sericea, Ximenia americana, Zanthoxylum humile
\end{tabular}

Distribution of the 53 medicinal plant species within different disease categories (Canhane, 2007-2008).

local rules to protect native plant species, particularly Adansonia digitata, B. discolor, Cissus cornifolia, C. mopane, E. elephantina, F. sycomorus, F. virosa, G. monticola, G. conjugata, Manilkara mochisia, S. birrea, and Strychnos madagascariensis. Other conservation measures included community guards in protected places to control fires and logging, mostly due to South African migrants. On the other hand, trading was controlled and confined to the village.

\section{Conclusions}

This study shows the social importance of the floristic richness in the Canhane village, particularly regarding the significance of medicinal plants in primary healthcare. This is reflected in the great diversity of plants used for medical purposes as well as in the wide range of their applications and associated procedures. The data compiled in this study are a contribution to the documentation of PGR at the national and regional level 
Table 3 List of medicinal plants (25 spp.) surveyed in the district of Massingir in 1960-70

\begin{tabular}{|c|c|c|}
\hline Scientific name* & Local name & Diseases- Gaza district \\
\hline \multicolumn{3}{|l|}{ Alismataceae } \\
\hline $\begin{array}{l}\text { Limnophyton obtusifolium (L.) } \\
\text { Miq. }\end{array}$ & NA & Ear diseases \\
\hline \multicolumn{3}{|l|}{ Amaranthaceae } \\
\hline Chenopodium ambrosioides L. & Kanunka uncono & Intestinal ulcers; Stomach-aches \\
\hline $\begin{array}{l}\text { Hermbstaedtia odorata (Burch.) } \\
\text { T. Cooke }\end{array}$ & Chomeli & Diuretics; Stomach wash \\
\hline \multicolumn{3}{|l|}{ Anacardiaceae } \\
\hline $\begin{array}{l}\text { Lannea schweinfurthii (Engl.) } \\
\text { Engl. }\end{array}$ & $\begin{array}{l}\text { chiumbocanhe, chebombocanho, } \\
\text { munganicomo }\end{array}$ & Abdominal pain; Choleric diarrhea; Cough; Tuberculosis \\
\hline $\begin{array}{l}\text { Ozoroa obovata (Oliv.) R.Fern. \& } \\
\text { A. Fern. }\end{array}$ & $\begin{array}{l}\text { xinungu, chimungumango, chinungo, } \\
\text { chinungumafe, chinungumafi }\end{array}$ & Diarrhea; Laxative; Pain \\
\hline Sclerocarya birrea Sond. & $\begin{array}{l}\text { canhi (tree and fruit), tsula (tree), ditsula } \\
\text { (fruit) }\end{array}$ & Diarrhea; dysentery \\
\hline \multicolumn{3}{|l|}{ Apocynaceae } \\
\hline Adenium multiflorum Klotzsch & chimua & Male sterility; Sexual performance \\
\hline $\begin{array}{l}\text { Pergularia daemia (Forssk.) } \\
\text { Chiov. }\end{array}$ & furana & Antiemetic; Cough \\
\hline
\end{tabular}

Chiov.

\section{Araceae}

\begin{tabular}{|c|c|c|}
\hline Stylochiton natalensis Schott & NA & Ear diseases; Respiratory diseases; Tranquilizing \\
\hline \multicolumn{3}{|l|}{ Asteraceae } \\
\hline Ageratum conyzoides $\mathrm{L}$. & NA & Abdominal disorders; Laxative \\
\hline \multicolumn{3}{|l|}{ Burseraceae } \\
\hline $\begin{array}{l}\text { Commiphora africana (A. Rich.) } \\
\text { Engl. }\end{array}$ & NA & Abdominal disorders; Asthma; Head ache; Stomach ache \\
\hline \multicolumn{3}{|l|}{ Capparaceae } \\
\hline Boscia mossambicensis Klotzsch & Chimapamapane, chicutlu & Eye disinfectant \\
\hline Cadaba natalensis Sond. & tssatssassana, mejacocone & Tuberculosis \\
\hline Capparis tomentosa Lam. & caua, cahu & Respiratory diseases; Tuberculosis \\
\hline $\begin{array}{l}\text { Cladostemon kirkii (Oliv.) Pax \& } \\
\text { Gilg }\end{array}$ & $\begin{array}{l}\text { tumangoma, mahuco, maúco, buguane, } \\
\text { tambocolata }\end{array}$ & Abdominal disorders; Colds; Sexual performance; Venereal diseases \\
\hline Thilachium africanum Lour. & compfa, compha & Asthma; Diarrhoea; Edema; General pain; Vomiting \\
\hline \multicolumn{3}{|l|}{ Celastraceae } \\
\hline $\begin{array}{l}\text { Elaeodendron schlechteranum } \\
\text { (Loes.) Loes. }\end{array}$ & chigugutzo; chigugusse & Aphrodisiac; Deworming. \\
\hline $\begin{array}{l}\text { Maytenus senegalensis (Lam.) } \\
\text { Exell }\end{array}$ & Chixangua, Chichangua; chilhangua & $\begin{array}{l}\text { Bilharziosis; Bronchitis and tuberculosis; Convulsions; Diarrhoea and } \\
\text { dysentery; Male and female sterility }\end{array}$ \\
\hline \multicolumn{3}{|l|}{ Combretaceae } \\
\hline Combretum apiculatum Sond. & Chivonzôane, samabulile & Abdominal pain; Conjunctivitis \\
\hline Combretum imberbe Wawra & Monzou; mondzo & Bilharziosis; Stomach-aches \\
\hline $\begin{array}{l}\text { Combretum microphyllum } \\
\text { Klotzsch }\end{array}$ & Funté, mumbambanguene pfunte & Abdominal pain; Bilharziosis; Diarrhoea; Female sterility \\
\hline $\begin{array}{l}\text { Combretum molle R.Br ex G. } \\
\text { Don }\end{array}$ & Chicucudze, xicucutce & Antiabortifacients, Dysentery \\
\hline $\begin{array}{l}\text { Combretum mossambicense } \\
\text { (Klotzsch) Engl. }\end{array}$ & Futé, funté, fute & Diarrhoea; Laxative \\
\hline Combretum zeyheri Sond. & NA & Eye cleaning \\
\hline $\begin{array}{l}\text { Pteleopsis myrtifolia (M.A. } \\
\text { Lawson) Engl. \& Diels }\end{array}$ & Ludzane & Fever; Madness Male and female sterility \\
\hline
\end{tabular}

\section{NA = Not Available}

The table provides the family, scientific and local names, habit and diseases of $25 \mathrm{spp}$. surveyed in the district of Massingir mainly during the $60-70 \mathrm{~s} .{ }^{*}[11,12,43]$ 
Table 4 Other applications of the surveyed plant species from Canhane village (2007- 2008)

Scientific name

Acanthaceae

Blepharis diversispina (Nees) C.B. Clarke

Aloaceae

Aloe marlothii A. Berger

Aloe zebrina Baker

\section{Anacardiaceae}

Lannea schweinfurthii (Engl.) Engl.

Sclerocarya birrea Hochst.

\section{Apocynaceae}

Sarcostemma viminale (L.) R.Br.

Asparagaceae

Asparagus africanus Lam.

Balanitaceae

Balanites maughamii Sprague

\section{Bombacaceae}

Adansonia digitata $\mathrm{L}$.

\section{Capparaceae}

Boscia albitrunca (Burch.) Gilg \& Gilg-Ben.

Maerua edulis (Gilg \& Gilg-Ben.) DeWolf

Maerua parvifolia Pax

\section{Celastraceae}

Loeseneriella crenata (Klotzsch) Wilczek ex N. Hallé

\section{Combretaceae}

Combretum imberbe Wawra

Terminalia sericea Burch. ex DC

\section{Cucurbitaceae}

Cucumis zeyheri Sond.

Cucumis metuliferus E.Mey. ex Naudin

\section{Dracaenaceae}

Sansevieria hyacinthoides (L.) Druce

\section{Ebenaceae}

Euclea racemosa Murr.

\section{Euphorbiaceae}

Androstachys johnsonii Prain

Flueggea virosa (Roxb. ex Willd.) Voigt

Spirostachys africana Sond.

\section{Fabaceae}

Cassia abbreviata Oliv.

Colophospermum mopane (Benth.) Léonard

Dalbergia melanoxylon Guill. \& Perr

Dichrostachys cinerea (L.) Wight \& Arn

Elephantorrhiza elephantina (Burch.) Skeels

Guibourtia conjugata (Bolle) J.Léonard

\section{Malvaceae}

Gossypium herbaceum L.

Hibiscus meyeri Harv.

\section{Other Applications}

Beliefs and myths

Beliefs and myths; Food (nectar); Veterinary (cattle diseases)

Food (leaves); Veterinary (cattle, chicken and lamb's diseases)

Beliefs and myths; Food (fruits); Handicraft, Ornamental

Beliefs and myths; Food (fruits); Handicraft; Ornamental; Wood

Food (fruits); Forage

Beliefs and myths

Beliefs and myths; Forage; Ornamental; Wood

Food (fruits); Paper

Food (fruits); Firewood; Handicraft; Wood

Food (Fruits)

Beliefs and myths; Fishery; Food (Fruits)

Beliefs and myths; Carts and animal traction; Textile

Charcoal; Firewood; Kitchen handicraft; Wood

Beliefs and myths; Fiber; Firewood; Handicraft; Ornamental; Wood

Beliefs and myths; Food (leaves); Veterinary (Cattle and lambs wounds)

Veterinary (Goat diseases, Newcastle disease)

Beliefs and myths; Textile

Cosmetics; Food (fruits)

Wood

Beliefs and myths; Food (fruits)

Veterinary (cattle eye diseases); Wood

Beliefs and myths; Ornamental; Wood

Charcoal; Firewood; Handicraft; Wood

Handicraft; Musical instruments

Beliefs and myths; Farm fencing; Forage

Beliefs and myths

Beliefs and myths; Firewood; Glue; Handicraft; Ornamental

Food (Leaves); Textile

Aphrodisiac; Broom 
Table 4: Other applications of the surveyed plant species from Canhane village (2007- 2008) (Continued)

\begin{tabular}{|c|c|}
\hline \multicolumn{2}{|l|}{ Meliaceae } \\
\hline Trichilia emetica Vahl subsp. emetica & Cosmetics; Food (fruits, seeds) \\
\hline \multicolumn{2}{|l|}{ Moraceae } \\
\hline Ficus sycomorus $\mathrm{L}$. & Beliefs and myths; Food (fruits); Forage \\
\hline \multicolumn{2}{|l|}{ Olacaceae } \\
\hline Olax dissitiflora Oliv. & Beliefs and myths; Food (fruits); Forage; Wood \\
\hline Ximenia americana L. & Beliefs and myths; Cosmetics; Farm fencing; Food (fruits, seeds); Veterinary (cattle wounds) \\
\hline \multicolumn{2}{|l|}{ Orchidaceae } \\
\hline Ansellia africana Lindl. & Beliefs and myths \\
\hline \multicolumn{2}{|l|}{ Poaceae } \\
\hline Cynodon dactylon (L.) Pers. & Forage \\
\hline \multicolumn{2}{|l|}{ Rubiaceae } \\
\hline Gardenia volkensii K. Schum. & Beliefs and myths; Food (fruits); Textile \\
\hline \multicolumn{2}{|l|}{ Rutaceae } \\
\hline Zanthoxylum humile (E.A. Bruce) P.G. Waterman & Beliefs and myths; Snake repellent \\
\hline \multicolumn{2}{|l|}{ Sapotaceae } \\
\hline Manilkara mochisia (Baker) Dubard & Food (fruits) \\
\hline \multicolumn{2}{|l|}{ Strychnaceae } \\
\hline Strychnos madagascariensis Spreng. ex Baker & Beliefs and myths; Food (fruits); Handicrafts; Musical instruments \\
\hline \multicolumn{2}{|l|}{ Sterculiaceae } \\
\hline Hermannia micropetala Harv. \& Sond. & Beliefs and myths \\
\hline \multicolumn{2}{|l|}{ Tiliaceae } \\
\hline Grewia flavescens Juss. var. flavescens & Beliefs and myths; Food (fruits) \\
\hline Grewia hexamita Burret & Food (fruits); Handicraft \\
\hline Grewia monticola Sond. & Food (fruits); Ornamental; Veterinary (relieves cow's pain during calf-birth) \\
\hline \multicolumn{2}{|l|}{ Vitaceae } \\
\hline Cissus cornifolia (Bak.) Planch. & Food (fruits); Repellent; Veterinary (cattle wounds) \\
\hline Cissus quadrangularis $L$. & Beliefs and myths; Repellent; Veterinary (cattle Newcastle disease, wounds) \\
\hline
\end{tabular}

The table presents a list of $46 \mathrm{spp}$. which, besides their medicinal use, are used for non-medical purposes (e.g. applications related to beliefs and myths, food, handicraft, animal diseases, ornamental).

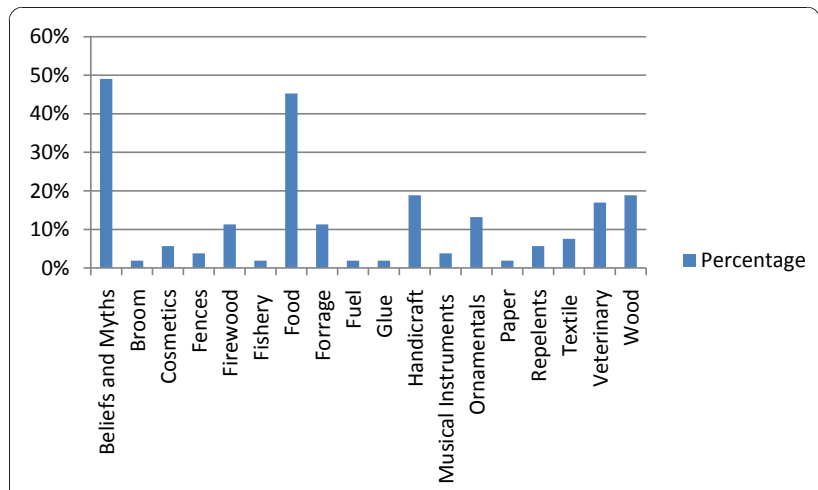

Figure 2 Non-medical applications. Non-medical applications of the 53 medicinal plant species (Canhane, 2007- 2008).

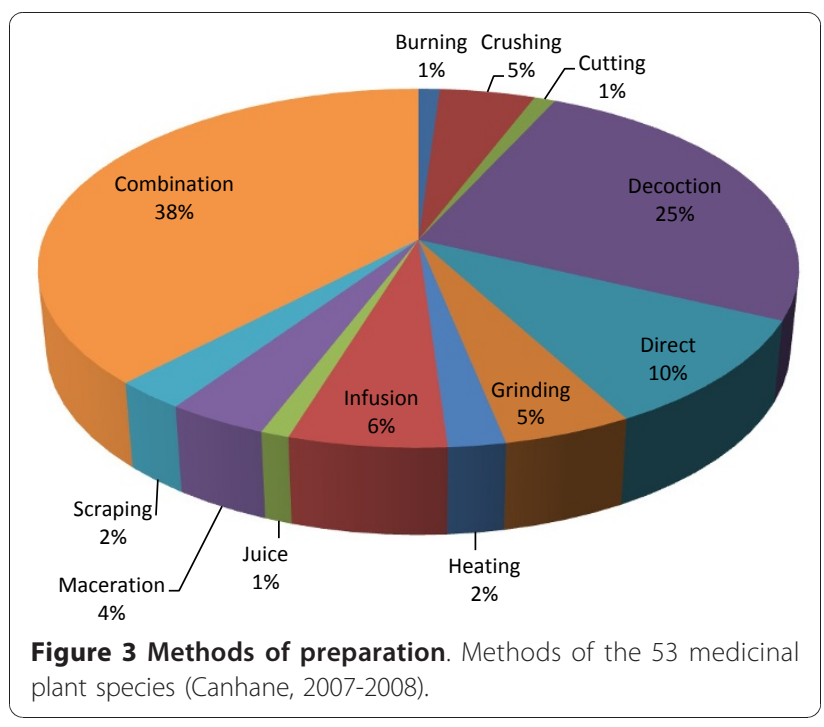




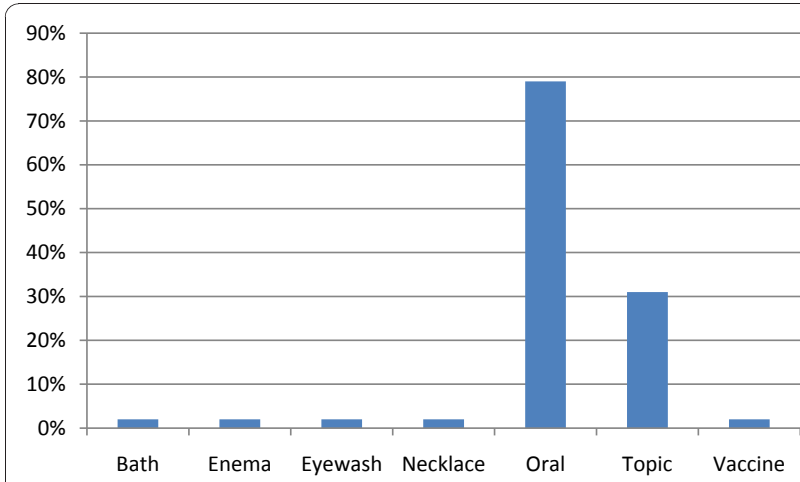

Figure 4 Administration routes. Administration routes of the 53 medicinal plant species (Canhane, 2007-2008).

and can serve as a basis to develop larger and interdisciplinary studies.

\section{List of abbreviations}

GLTP: Great Limpopo Transfrontier Park and Conservation Area; PGR: Plant Genetic Resources.

\section{Acknowledgements}

The authors would like to express their gratitude to the Canhane community, particularly the 11 informants for their unreserved efforts in transmitting traditional local knowledge, the Canhane Community Lodge for coordinating the work with the community and for the logistics, the direction of Paulo Samuel Kankhomba Primary school for selecting the students and Non-Governamental Organization LUPA. Acknowledgments are also due to botanical collectors, António Zacarias and Ernesto Macamo, and translators, Arminda Mfumo, dr. David Langa and dr. Orlando Bahule. Thanks to Dr. José Manuel Mota Cardoso (Veterinary Hospital, Eduardo Mondlane University, Mozambique) for medical terminology and language revision, Dr. Katharina Pawlowsi (Stockholm University) for the language revision, Dr. Cristina Duarte (Tropical Research Institute, Portugal) for the taxonomic revision, and Ezequiel Correia for preparing the distribution map of the study area. This work was supported by a grant from Fundo Aberto Universidade Eduardo Mondlane and the Swedish International Development Agency (Research Funding).

\section{Author details}

${ }^{1}$ Tropical Research Institute, Rua da Junqueira 86, 1300-344, Lisbon, Portugal. ${ }^{2}$ Faculdade de Agronomia e Engenharia Florestal, Universidade Eduardo Mondlane, CP 257, Maputo, Mozambique.

\section{Authors' contributions}

The design, planning, field survey and taxonomic analysis was coordinated and conducted by TF. AR and TF performed the data processing and analysis. The taxonomic revision was done by MMR and JT. Data from other geographical regions and from 1960-70 was retrieved by MMR, JT and TF. Literature retrieval was done by AR and MMR. AR wrote the manuscript, which was revised by MMR and TF. All authors read and approved the manuscript.

\section{Competing interests}

The authors declare that they have no competing interests.

Received: 18 June 2010 Accepted: 3 December 2010 Published: 3 December 2010

\section{References}

1. Hamilton AC: Medicinal plants, conservation and livelihoods. Biodiver Conserv 2004, 13:1477-1517.
2. Hiremath VT, Taranath TC: Traditional phytotherapy for snake bites by tribes of Chitradurga District, Karnataka, India. Ethnobot Leaflets 2010, 14:120-25.

3. World Health Organization: WHO traditional medicine strategy 2002-2005. World Health Organization; 2002.

4. de Vicente MC, Guzmán FA, Engels J, Rao VA: Genetic characterization and its use in decision-making for the conservation of crop germplasm. In The role of biotechnology in exploring and protecting agricultural genetic resources. Edited by: Ruane J, Sonnino A. FAO of the United Nations: 2006:2129-138

5. Fraleigh B: Global overview of crop genetic resources. In The role of biotechnology in exploring and protecting agricultural genetic resources. Edited by: Ruane J, Sonnino A. FAO of the United Nations; 2006:21-32.

6. Gepts P: Plant genetic resources conservation and utilization: the accomplishments and future of a societal insurance policy. Crop Sci 2006, 46:2278-2292.

7. Krog M, Falcão MP, Olsen CS: Medicinal plant markets and trade in Maputo, Mozambique. Forest \& Landscape Working Papers 16, Danish Center for forest landscaping and planning, KVL 2006.

8. Jansen PCM, Mendes O: Plantas medicinais - Seu uso traditional em Moçambique. Tomo 3. Ministério da Saúde, Maputo 1990.

9. Chamba $E$, Mangue $P$ : The role of traditional structures in conservation of natural resources: Potone case study. 3rd Regional Workshop of CASS/ PLASS Community based natural resource management programme, Maputo 2001.

10. Bandeira SO, Gaspar F, Pagula FP: African ethnobotany and healthcare: Emphasis on Mozambique. Pharm Biol 2001, 39:70-73.

11. Watt JM, Breyer-Brandwijk MG: Medicinal and poisonous plants of Southern and Eastern Africa. Edinburg and London: E \& S Livingstone Ltd; 1962.

12. Faria MT: Revisão de algumas Combretaceae de Moçambique. Mem Inst Inv Agron Moç 1973, 4:37-45.

13. Sitoe A, Chaúque A: Plano de Gestão dos Recursos Naturais da Comunidade de Canhane, Distrito de Massingir. Relatório de Consultoria. Lupa, Associação para o Desenvolvimento da Comunidade 2008.

14. Ministério da Administração Estatal: O perfil do distrito de Massingir. Série: Perfis distritais 2005.

15. Reddy SJ: Agroclimate of Mozambique as relevant to dry-land agriculture. Instituto de Investigação Agronómica de Moçambique 2008

16. HELVETAS, Moçambique: Projecto de Desenvolvimento de Turismo baseado na Comunidade de Canhane, Distrito de Massingir-Gaza (Área de Conservação Transfronteira do Grande Limpopo). 2002.

17. Faria T: Etnobotânica da aldeia de Canhane. Plantas úteis, saberes locais e tradições. Maputo: Diname Pub; 2010.

18. Gomes PJ, Barbosa LAG: Esboço do Reconhecimento Ecológico-Agrícola de Moçambique. Lourenço Marques. Mem Trab CICA 1955, 23:67-226.

19. Wild H, Barbosa LAG: Vegetation Map of the Flora Zambesiaca Area. Flora Zambesiaca Supplement Salisbury: M. O. Collins (Pvt) Ltd; 1968.

20. Martin GJ: Ethnobotany: A Methods Manual. London: Chapman and Hall: 1995.

21. Cotton CM: Ethnobotany: Principles and Applications. Chichester: John Wiley and Sons Ltd; 1996.

22. Van Wyk B: A Photographic Guide to Wild Flowers of South Africa. Cape Town: Struik Publishers (PTY) Ltd; 2000.

23. Van Wyk P: A Photographic Guide to Trees of Southern Africa. Cape Town: Struik Publishers (PTY) Ltd; 2001.

24. Van Wyk P: Field Guide to Trees of the Kruger National Park. Cape Town: Struik Publishers (PTY) Ltd; 2001.

25. Van Wyk B, Van Wyk P: Field Guide to Trees of Southern Africa. Cape Town: Struik Publishers (PTY) Ltd; 2001.

26. African Plants Database. [http://www.ville-ge.ch/musinfo/bd/cjb/africa/]

27. Tropicos.org. Missouri Botanical Garden. [http://www.tropicos.org].

28. The International Plant Names Index. [http://www.ipni.org].

29. Simbo D: An ethnobotanical survey of medicinal plants in Babungo, Northwest Region, Cameroon. J Ethnobiol Ethnomed 2010, 6:8.

30. Mesfin F, Demissew S, Teklehaymanot T: An ethnobotanical study of medicinal plants in Wonago Woreda, SNNPR, Ethiopia. J Ethnobiol Ethnomed 2009, 5:28.

31. Bekalo TH, Woodmatas SD, Woldemariam ZA: An ethnobotanical study of medicinal plants used by local people in the lowlands of Konta Special 
Woreda, southern nations, nationalities and peoples regional state, Ethiopia. J Ethnobiol Ethnomed 2009, 5:26.

32. Giday M, Asfaw Z, Woldu Z, Teklehaymanot T: Medicinal plant knowledge of the Bench ethnic group of Ethiopia: an ethnobotanical investigation. J Ethnobiol Ethnomed 2009, 5:34.

33. Panghal M, Arya V, Yadav S, Kumar S, Yadav P: Indigenous knowledge of medicinal plants used by Saperas community of Khetawas, Jhajjar District, Haryana, India. J Ethnobiol Ethnomed 2010, 6:4.

34. Long C, Li S, Long B, Shi1 Y, Liu5 B: Medicinal plants used by the Yi ethnic group: a case study in central Yunnan. J Ethnobiol Ethnomed 2009, $5 \cdot 13$.

35. Bussmann RW, Sharon D: Traditional medicinal plant use in Northern Peru: tracking two thousand years of healing culture. J Ethnobiol Ethnomed 2006, 2:47.

36. Asres K, Bucar F, Kartnig T, Witvrouw M, Pannecouque C, De Clercq E: Antiviral Activity Against Human Immunodeficiency Virus Type 1 (HIV-1) and Type 2 (HIV-2) of Ethnobotanically Selected Ethiopian Medicinal Plants. Phytother Res 2001, 15:62-69.

37. Gathirwa JW, Rukunga GM, Njagi EN, Omar SA, Mwitari PG, Guantai AN, Tolo FM, Kimani CW, Muthaura CN, Kirira PG, Ndunda TN, Amalemba G, Mungai GM, Ndiege IO: The in vitro anti-plasmodial and in vivo antimalarial efficacy of combinations of some medicinal plants used traditionally for treatment of malaria by the Meru community in Kenya. J Ethnopharmacol 2008, 115:223-231.

38. Taylor JSL, Elgorashi EE, Maes A: Investigating the safety of plants used in South African traditional medicine: testing for genotoxicity in the micronucleus and alkaline content assays. Environ Mol Mutagenesis 2003, 42:144-154.

39. Innocent E, Moshi MJ, Masimbam PJ, Mbwambo ZH, Kapingu MC Kamuhabwa A: Screening of raditionally used Plants for in vivo antimalarial activity in mice. African J Trad Complem Altern 2009, 6:163-167.

40. Chinemanaa F, Drummondb RB, Mavib S, De Zoysac I: Indigenous plant remedies in Zimbabwe. J Ethnopharmacol 1985, 14:159-172.

41. Johns T, Faubert GM, Kokwaro JO, Mahunnah RLA, Kimanani EK: Antigiardial activity of gastrointestinal remedies of the Luo of East Africa. J Ethnopharmacol 1995, 46:17-23.

42. Galvez J, Zarzuelo A, Crespo ME, Utrilla MP, Jiménez J, Spiessens C, de Witte P: Antidiarrhoeic activity of Sclerocarya birrea bark extract and its active tannin constituent in rats. Phytother Res 2006, 5:276-278.

43. Ruffo CK: A Survey of medicinal plants in Tabora region, Tanzania. Traditional Medicinal Plants Dar Es Salaam: University Press, Ministry of Health; 1991.

44. Mathabe MC, Nikolova RV, Lall N, Nyazema NZ: Antibacterial activities of medicinal plants used for the treatment of diarrhoea in Limpopo Province, South Africa. J Ethnopharmacol 2006, 105:286-293.

45. Samie A, Obi CL, Bessong PO, Namrita L: Activity profiles of fourteen selected medicinal plants from Rural Venda communities in South Africa against fifteen clinical bacterial species. African J Biotechnol 2005, 4:1443-1451.

46. Kokwaro JO: Medicinal Plant of East Africa. Kampala, Nairobi, Dar es Salaam: East African Literature Bureau; 1976.

47. Jain A, Katewa SS, Galav PK, Sharma P: Medicinal plant diversity of Sitamata wildlife sanctuary, Rajasthan, India. J Ethnopharmacol 2005, 102:143-157.

48. Mmatli EE, Malerød H, Wilson SR, Abegaz B, Greibrokk T, Lundanes E, Malterud KE, Petersen D, Rise F: Identification of major metal complexing compounds in Blepharis aspera. Anal Chim Acta 2007, 597:24-31.

49. Nanyingi MO, Mbaria JM, Lanyasunya AL, Wagate CG, Koros KB, Kaburia HF, Munenge RW, Ogara WO: Ethnopharmacological survey of Samburu district, Kenya. J Ethnobiol Ethnomed 2008, 4:14.

doi:10.1186/1746-4269-6-33

Cite this article as: Ribeiro et al:. Ethnobotanical survey in Canhane village, district of Massingir, Mozambique: medicinal plants and traditional knowledge. Journal of Ethnobiology and Ethnomedicine 2010 6:33.

\section{Submit your next manuscript to BioMed Central and take full advantage of:}

- Convenient online submission

- Thorough peer review

- No space constraints or color figure charges

- Immediate publication on acceptance

- Inclusion in PubMed, CAS, Scopus and Google Scholar

- Research which is freely available for redistribution 